\title{
Tratamento de semente e aplicação foliar de fungicidas para o controle da ferrugem-da-soja
}

\author{
Cláudia Vieira Godoy(1) e Ademir Assis Henning ${ }^{(1)}$ \\ (1)Embrapa Soja, Caixa Postal 231, CEP 86001-970 Londrina, PR. E-mail: godoy@cnpso.embrapa.br, henning@cnpso.embrapa.br
}

\begin{abstract}
Resumo - O objetivo deste trabalho foi avaliar a eficiência do tratamento de semente de soja com fluquinconazol e da aplicação foliar de fungicidas no manejo da ferrugem-da-soja, em três épocas de semeadura, na safra 2007/2008, em Londrina, PR. O delineamento foi o de blocos ao acaso, em arranjo fatorial 2x5, com dois tratamentos de semente com fluquinconazol e cinco aplicações de misturas de fungicidas na primeira época de semeadura. Na segunda e na terceira época o arranjo fatorial foi de 2x6. Avaliou-se a área abaixo da curva de progresso da doença, o índice vegetativo por diferença normalizada (NDVI) e a produtividade. Não se verificou interação significativa entre o tratamento de semente com fluquinconazol e os tratamentos de mistura de fungicidas aplicados na parte aérea. O tratamento de semente com fluquinconazol não atrasou a evolução da doença e não apresentou diferença significativa com o tratamento sem fluquinconazol, em nenhuma das épocas e variáveis analisadas. O NDVI apresentou alta correlação com as avaliações de severidade e produtividade, em todas as épocas de semeadura. A resposta em produtividade, em conseqüência da época e do intervalo de aplicação, variou de acordo com a época de semeadura e com o momento da incidência da doença.
\end{abstract}

Termos para indexação: Phakopsora pachyrhizi, controle, fluquinconazol.

\section{Seed treatment and foliar fungicide application to control soybean rust}

\begin{abstract}
The objective of this work was to evaluate the effect of soybean seed treatment with fluquinconazole and foliar fungicide application on soybean rust management, in three sowing dates, during the 2007/2008 growing season, in Londrina, PR, Brazil. The experimental design was a randomized complete block, with a factorial arrangement of $2 \times 5$ (with two treatments of seeds with fluquinconazole, and five foliar fungicide applications) in the first sowing time, and of $2 \times 6$ in the second and third sowing time. The parameters evaluated were: the area under disease progress curve, the normalized difference vegetation index (NDVI) and yield. There was no significant interaction between seed treatment with fluquinconazole and fungicide mixture in foliar applications. Seed treatment with fluquinconazole did not delay the disease progress, and showed no significant difference from treatment without fluquinconazole for sowing time or any other parameter evaluated. The NDVI showed high correlation with disease severity and yield, in all sowing times. Yield response for time and the interval of application varied according to sowing dates and the time of disease onset.
\end{abstract}

Index terms: Phakopsora pachyrhizi, control, fluquinconazole.

\section{Introdução}

A ferrugem-asiática-da-soja (FAS), causada pelo fungo Phakopsora pachyrhizi Syd. \& P. Syd., é uma das doenças mais severas para a cultura da soja [Glycine max (L.) Merr.], com relatos de danos de 10 a 90\% (Sinclair \& Hartman, 1999). Do continente africano, na década de 1990 (Levy, 2005), o fungo se disseminou para a América do Sul, onde foi detectado em 2001 no Paraguai e no Sul do Brasil (Yorinori et al., 2005). O custo da FAS no Brasil, desde as primeiras epidemias severas até a safra de 2006/2007, foi estimado em aproximadamente 7,7 bilhões de dólares americanos, incluídas as perdas em produção e o custo com o controle dessa doença.
Os sintomas iniciais da doença são pequenas pústulas foliares, de coloração castanha a marromescura. Na face inferior da folha, pode-se observar uma ou mais urédias que se rompem e liberam os uredósporos. As lesões tendem ao formato angular e podem atingir 2 a $5 \mathrm{~mm}$ de diâmetro, em pecíolos, vagens e caules (Sinclair \& Hartman, 1999). Plantas severamente infectadas apresentam desfolha precoce, que compromete a formação e o enchimento de vagens e o peso final do grão. Quanto mais cedo ocorrer a desfolha, menor será o tamanho do grão e, conseqüentemente, maior a perda de rendimento e de qualidade (Yang et al., 1991).

As estratégias de manejo, recomendadas no Brasil, são: a utilização de cultivares de ciclo precoce e 
semeaduras no início da época recomendada; a eliminação de plantas de soja voluntárias e a ausência de cultivo de soja na entressafra; o monitoramento da lavoura desde o início do desenvolvimento da cultura; e a utilização preventiva de fungicidas ou no aparecimento dos sintomas. O tratamento de semente com fluquinconazol passou a ser recomendado em 2007 como mais uma estratégia de manejo da ferrugem-asiática e pode retardar a evolução da doença (Tecnologias..., 2008).

$\mathrm{O}$ tratamento de semente com fungicidas, na cultura da soja, é uma prática recomendada com o objetivo de controlar patógenos importantes e assegurar populações adequadas de plantas. Quando as condições edafoclimáticas durante a semeadura são desfavoráveis à germinação e à rápida emergência da soja, a semente fica exposta por mais tempo a fungos habitantes do solo, que podem causar a sua deterioração ou a morte de plântulas (Henning, 2005). O tratamento de semente padrão deve ser realizado com produtos de contato e sistêmicos. O fluquinconazol não é recomendado para o tratamento padrão, e sua recomendação é específica para atrasar a evolução da FAS na parte aérea (Tecnologias..., 2008).

$\mathrm{O}$ controle de doenças fúngicas da parte aérea, por meio do tratamento de semente é uma prática de manejo utilizada na cultura do trigo e permite atrasar a incidência de doenças como a ferrugem-da-folha (Puccinia triticina Erickss.), a mancha-bronzeada-dafolha [Drechslera tritici-repentis (Died.) Shoemaker] e o oídio [Blumeria graminis (DC) E.O. Speer f. sp. tritici] (Goulart, 1999; Picinini \& Fernandes, 2003).

Em trigo, embora o tratamento de semente cause atraso à evolução de diversas doenças, nem sempre esses resultados se refletem na produtividade, caso não seja realizado controle por meio de pulverizações na parte aérea, em razão da incidência tardia de algumas doenças, o que pode mascarar os resultados obtidos pelo tratamento de semente. Nesses casos, a avaliação do tratamento de semente é recomendada dentro de um sistema de manejo da doença (Goulart, 1999).

O presente trabalho teve como objetivo avaliar a eficiência do tratamento de semente de soja com fluquinconazol, no manejo da ferrugem-asiática-dasoja, em diferentes épocas de semeadura, associado a diferentes épocas e intervalos de aplicação de fungicidas na parte aérea.

\section{Material e Métodos}

O experimento foi conduzido na Embrapa Soja, em Londrina, PR, na safra 2007/2008. Foram realizadas semeaduras em três épocas, com as cultivares: BRS 255RR, de ciclo semi-precoce, semeada em 30/10/2007, e BRS 247RR, de ciclo médio, semeada em 22/11/2007 e 13/12/2007.

O delineamento foi o de blocos ao acaso, com os tratamentos em arranjo fatorial $2 \times 5$, com dois tratamentos de semente e cinco tratamentos na parte aérea, na primeira época de semeadura, e tratamentos similares em arranjos $2 \times 6$, na segunda e na terceira épocas de semeadura, com quatro repetições; cada repetição foi constituída de parcelas com seis linhas de plantas, espaçadas em $0,45 \mathrm{~m}$, e com $6 \mathrm{~m}$ de comprimento.

Toda a semente foi tratada com carbendazin + thiram $(30+70 \mathrm{~g}$ de i.a. por $100 \mathrm{~kg}$ sementes), conforme o padrão de tratamento, e depois metade foi tratada com fluquinconazol, na dose $50 \mathrm{~g}$ de i.a. por $100 \mathrm{~kg}$ de sementes, recomendado especificamente para FAS. Foi adicionada água, para completar o volume final de calda para $500 \mathrm{~mL}$ por $100 \mathrm{~kg}$ de sementes, nos tratamentos que receberam apenas o tratamento com o fungicida padrão.

Na primeira época de semeadura, nos tratamentos da parte aérea, foram aplicadas as misturas de: piraclostrobina + epoxiconazol e trifloxistrobina + tebuconazol, em R2 e em R5 (T2 e T3); trifloxistrobina + tebuconazol somente em R2 (T4) e somente em R5 (T5). $\mathrm{Na}$ segunda e na terceira épocas de semeadura, nos tratamentos da parte aérea, foram aplicadas as misturas de: piraclostrobina + epoxiconazol e trifloxistrobina + tebuconazol, no início dos sintomas e 20 dias após a primeira aplicação (DAA1) (T2 e T3); trifloxistrobina + tebuconazol, no momento em que a doença atingiu $1 \%$ de severidade, e aos 20 DAA1 (T4) ou 30 DAA1 (T6); e trifloxistrobina + tebuconazol, no início dos sintomas e aos 30 DAA1 (T5). Na terceira época de semeadura, os tratamentos 2,3 e 4 receberam uma terceira aplicação, 20 dias após a segunda, em razão do início precoce da doença. Os estados fenológicos adotados no presente trabalho seguiram a descrição de Ritchie et al. (1985).

A dose de piraclostrobina + epoxiconazol, utilizada nos ensaios, foi de $66,5+25 \mathrm{~g} \mathrm{ha}^{-1}$ de i.a. e a de trifloxistrobina + tebuconazol foi de $50+100 \mathrm{~g} \mathrm{ha}^{-1}$ de i.a. acrescida de adjuvante $0,25 \% \mathrm{v} / \mathrm{v}$. As aplicações 
foram realizadas com pulverizador costal, pressurizado com $\mathrm{CO}_{2}$, equipado com barra de $2 \mathrm{~m}$, com quatro bicos Teejet XR 110:02, espaçados de $50 \mathrm{~cm}$, e calibrada para vazão de $200 \mathrm{~L} \mathrm{ha}^{-1}$. As aplicações de fungicidas foram realizadas no período da manhã, com umidade relativa acima de $60 \%$ e temperatura abaixo de $30^{\circ} \mathrm{C}$.

A média de severidade das parcelas (área foliar coberta com sintomas) foi estimada visualmente, com auxílio de escala diagramática (Godoy et al., 2006). Foram avaliadas as folhas nos terços inferior, médio e superior de quatro pontos na parcela, semanalmente, a partir dos primeiros sintomas, para o cálculo da área abaixo da curva de progresso da doença (AACPD) (Campbell \& Madden, 1990).

Foram realizadas avaliações, por meio de sensoriamento remoto, do índice de vegetação por diferença normalizada (NDVI - "normalized difference vegetation index") (Nilsson, 1995), quando a ferrugem atingiu o dossel superior dos tratamentos, tendo-se utilizado o equipamento Greenseeker.

A colheita foi realizada com colhedora de parcelas Wintersteiger modelo classic. A produtividade foi calculada em quilogramas por hectare, a $13 \%$ de umidade. As análises dos resultados foram realizadas pelo teste de comparações múltiplas de médias de Tukey, a $5 \%$ de probabilidade, com o SAS (SAS Institute, 2001).

\section{Resultados e Discussão}

O estádio fenológico em que foram observados os primeiros sintomas da FAS variou de acordo com a época de semeadura. Não houve resposta significativa nas épocas de semeadura quanto à interação entre o tratamento de semente com fluquinconazol e os tratamentos na parte aérea. $\mathrm{O}$ efeito do tratamento de semente com fluquinconazol não foi significativo, tendo sido apresentado somente o teste de comparações múltiplas de médias de Tukey $(\mathrm{p}<0,05)$ quanto aos tratamentos da parte aérea.

$\mathrm{Na}$ primeira semeadura, os primeiros sintomas foram observados em R4. Os tratamentos com duas aplicações das misturas piraclostrobina + epoxiconazol (T2) e trifloxistrobina + tebuconazol (T3) foram estatisticamente semelhantes, em todas variáveis analisadas (Tabela 1). O tratamento com aplicação única, realizada em R2 (T4), antes do início dos sintomas, apresentou residual insuficiente para o controle da doença, até o enchimento de grão, de acordo com as avaliações de severidade realizadas em R5.4 e R5.5, em que a severidade média estimada foi superior ao tratamento com duas aplicações e se refletiu no maior valor da AACPD. $\mathrm{O}$ tratamento com aplicação única, realizada em R5 (T5), quando já havia sintomas nas plantas, apresentou severidade superior aos tratamentos com duas aplicações, porém, com valor de AACPD menor do que a aplicação única realizada em R2 (T4).Aaplicaçãoem R5 foirealizada com $1 \%$ de média de severidade na parcela, e o aumento expressivo da severidade, mesmo após a aplicação do fungicida, pode ser explicado pela existência de lesões incubadas nas plantas. Esse mesmo comportamento foi observado no trabalho de Godoy \& Canteri (2004), em que fungicidas aplicados durante o período de incubação não impediram a manifestação dos sintomas. $\mathrm{Na}$ avaliação do NDVI, realizada em R6, os tratamentos que apresentaram os maiores valores, que refletiram o maior enfolhamento e menor presença de folhas com sintomas de ferrugem no dossel superior, foram aqueles com duas aplicações (T2 e T3) e o de aplicação única realizada em R2 (T4). A avaliação de NDVI em R6 apresentou alta correlação com a de produtividade $(\mathrm{r}=0,99)$ e a de severidade $(r=-0,99)$ realizadas na mesma data.

Tabela 1. Severidade de ferrugem (\%) em R5.4, R5.5 e R6, área abaixo da curva de progresso da doença (AACPD), índice vegetativo por diferença normalizada (NDVI) e produtividade $\left(\mathrm{kg} \mathrm{ha}^{-1}\right)$, dos diferentes tratamentos na cultivar BRS 255RR de ciclo semi-precoce, semeada em 30/10/2007(1).

\begin{tabular}{|c|c|c|c|c|c|c|c|}
\hline \multirow[t]{2}{*}{ Tratamento } & \multirow{2}{*}{$\begin{array}{c}\text { Estádio } \\
\text { da aplicação }\end{array}$} & \multicolumn{3}{|c|}{ Severidade $(\%)$} & \multirow[t]{2}{*}{ AACPD } & \multirow{2}{*}{$\begin{array}{c}\text { NDVI } \\
\text { R6 }\end{array}$} & \multirow{2}{*}{$\begin{array}{l}\text { Produtividade } \\
\left.\qquad \mathrm{kg} \mathrm{ha}^{-1}\right)\end{array}$} \\
\hline & & R5.4 & $\mathrm{R} 5.5$ & R6 & & & \\
\hline 1. Testemunha & & $56,8 \mathrm{a}$ & $47,1 \mathrm{a}$ & $80,3 \mathrm{a}$ & $1.079 \mathrm{a}$ & $0,55 \mathrm{c}$ & $1.449 \mathrm{c}$ \\
\hline 2. Piraclostrobina + epoxiconazol & R2 e R5 & $0,5 \mathrm{~d}$ & $5,0 \mathrm{dc}$ & $28,5 \mathrm{c}$ & 149d & $0,74 \mathrm{a}$ & $2.652 \mathrm{a}$ \\
\hline 3. Trifloxistrobina + tebuconazol & $\mathrm{R} 2$ e $\mathrm{R} 5$ & $0,3 \mathrm{~d}$ & $3,3 \mathrm{~d}$ & $22,4 \mathrm{c}$ & $102 \mathrm{~d}$ & $0,76 \mathrm{a}$ & $2.808 \mathrm{a}$ \\
\hline 4. Trifloxistrobina + tebuconazol & R2 & $16,5 b$ & $27,8 \mathrm{~b}$ & $29,3 \mathrm{c}$ & $500 \mathrm{~b}$ & $0,73 \mathrm{a}$ & $2.552 \mathrm{ab}$ \\
\hline 5. Trifloxistrobina + tebuconazol & R5 & $6,4 \mathrm{c}$ & $10,5 \mathrm{c}$ & $45,7 \mathrm{~b}$ & $271 \mathrm{c}$ & $0,65 b$ & $2.196 \mathrm{~b}$ \\
\hline $\mathrm{CV}(\%)$ & & 17,6 & 21,1 & 24,8 & 7,6 & 5,4 & 11,4 \\
\hline
\end{tabular}

${ }^{(1)}$ Médias seguidas por letras iguais, nas colunas, não diferem entre si pelo teste de Tukey, a 5\% de probabilidade. 
As maiores produtividades foram observadas nos tratamentos com duas aplicações (T2 e T3) e com aplicação única em R2 (T4). O atraso na aplicação, quando realizada com $1 \%$ de severidade, se refletiu em redução da produtividade, conforme observado no T5. Ensaios conduzidos no Zimbábue, com as cultivares Soprano e Sonata, tratadas comflusilazol + carbendazin, em diferentes datas e número de aplicações, mostraram que na maioria dos casos a aplicação única não evitou reduções de produtividade, com exceção daqueles em que foi posicionada de forma adequada. A época de aplicação se mostrou crítica, e as aplicações realizadas 10 dias antes ou após o momento ideal apresentaram redução de produtividade (Miles et al., 2003). No entanto, ensaios conduzidos no Sul do Paraná, com aplicações realizadas após o início da doença, com severidade entre 0,16 e $10 \%$, mostraram adequado controle da doença e manutenção da produtividade, principalmente com o uso de combinações de triazóis e estrobilurinas (Reis et al., 2007).

$\mathrm{Na}$ segunda época de semeadura, as primeiras aplicações foram realizadas no estádio R2, com $0,05 \%$ de severidade nas folhas inferiores. As aplicações feitas no momento em que a doença atingiu $1 \%$ de severidade foram realizadas em R3. Todos os tratamentos da parte aérea foram superiores à testemunha sem controle, em todas variáveis analisadas (Tabela 2). $\mathrm{Na}$ avaliação realizada em R5.2, os tratamentos realizados no início do aparecimento dos sintomas (T2, T3 e T5) apresentaram menores valores de severidade. No entanto, na avaliação em R5.3, todos os tratamentos foram estatisticamente semelhantes na avaliação da severidade e do NDVI. Em R5.4, os maiores valores de NDVI foram observados em todos os tratamentos com trifloxistrobina + tebuconazol, independentemente do momento da aplicação (T3, T4, T5 e T6). As avaliações do NDVI em R5.3 e R5.4 apresentaram altas correlações com a produtividade $(r=0,95$ e $r=0,94$, respectivamente).

As maiores produtividades foram observadas nos tratamentos que tiveram: aplicações no início dos sintomas e reaplicações aos 20 DAA1 (T2 e T3) ou aos 30 DAA1 (T5), e no tratamento iniciado com $1 \%$ de severidade da doença e reaplicação aos 20 DAA1 (T4). $\mathrm{O}$ atraso no início da aplicação ( $1 \%$ de severidade), associado ao aumento no intervalo de aplicação (30 DAA1) resultou em redução de produtividade (T6).

Na terceira época de semeadura, os sintomas iniciais foram observados em V6, em que foram realizadas as aplicações no início dos sintomas. Em razão da incidência precoce da doença, os tratamentos 2,3 e 4, com reaplicações aos 20 DAA1, receberam uma terceira aplicação 20 dias após a segunda. Na avaliação realizada em R5, as menores severidades foram observadas nos tratamentos com trifloxistrobina + epoxiconazol, aplicados no início dos sintomas e com duas aplicações seqüenciais após 20 DAA1 (T3), e nas aplicações com a doença a $1 \%$ de severidade e mais duas aplicações seqüenciais após 20 DAA1 (T4) ou somente uma aos 30 DAA1 (T6) (Tabela 3). Em R5.3, a menor severidade foi observada somente no tratamento com trifloxistrobina + tebuconazol, no início dos sintomas e mais duas aplicações seqüenciais em intervalos de 20 dias (T4). A explicação para a menor severidade nesse tratamento, comparado ao no início dos sintomas e reaplicações seqüenciais após 20 DAA1, pode ser atribuído ao residual do produto. Embora o controle no T4 tenha se iniciado com atraso, a $1 \%$ de severidade da doença, isto tornou necessárias as aplicações até

Tabela 2. Severidade de ferrugem (\%) em R5.2 e R5.3, área abaixo da curva de progresso da doença (AACPD), índice vegetativo por diferença normalizada (NDVI) e produtividade $\left(\mathrm{kg} \mathrm{ha}^{-1}\right)$, dos diferentes tratamentos aplicados no início dos sintomas (I), com 1\% de severidade e reaplicados 20 ou 30 dias após a primeira aplicação (DAA1), na cultivar BRS 247RR de ciclo médio, semeada em $22 / 11 / 2007^{(1)}$.

\begin{tabular}{|c|c|c|c|c|c|c|c|}
\hline \multirow[t]{2}{*}{ Tratamento } & \multirow{2}{*}{$\begin{array}{c}\text { Momento } \\
\text { da aplicação }\end{array}$} & \multicolumn{2}{|c|}{ Severidade $(\%)$} & \multirow[t]{2}{*}{ AACPD } & \multicolumn{2}{|c|}{ NDVI } & \multirow{2}{*}{$\begin{array}{l}\text { Produtividade } \\
\left(\mathrm{kg} \mathrm{ha}^{-1}\right)\end{array}$} \\
\hline & & R5.2 & R5.3 & & R5.3 & R5.4 & \\
\hline 1. Testemunha & & $30,4 a$ & $60,8 \mathrm{a}$ & $1.177 \mathrm{a}$ & $0,61 b$ & $0,47 \mathrm{c}$ & $618 \mathrm{c}$ \\
\hline 2. Piraclostrobina + epoxiconazol & I/20 DAA1 & $6,5 \mathrm{~d}$ & $28,4 b$ & $577 \mathrm{bc}$ & $0,74 \mathrm{a}$ & $0,65 b$ & $1.580 \mathrm{ab}$ \\
\hline 3. Trifloxistrobina + tebuconazol & I/20 DAA1 & $3,5 \mathrm{~d}$ & $29,1 b$ & $457 \mathrm{c}$ & $0,79 \mathrm{a}$ & $0,69 \mathrm{ab}$ & $1.881 \mathrm{a}$ \\
\hline 4. Trifloxistrobina + tebuconazol & $1 \% / 20$ DAA1 & $17,2 b$ & $33,6 b$ & $661 b$ & $0,77 \mathrm{a}$ & $0,73 \mathrm{a}$ & $1.697 \mathrm{ab}$ \\
\hline 5. Trifloxistrobina + tebuconazol & I/30 DAA1 & $8,2 \mathrm{~cd}$ & $30,3 b$ & $517 \mathrm{bc}$ & $0,76 \mathrm{a}$ & $0,68 \mathrm{ab}$ & $1.500 \mathrm{ab}$ \\
\hline 6. Trifloxistrobina + tebuconazol & $1 \% / 30$ DAA1 & $12,5 \mathrm{bc}$ & $24,3 b$ & $549 \mathrm{bc}$ & $0,78 \mathrm{a}$ & $0,67 \mathrm{ab}$ & $1.477 \mathrm{~b}$ \\
\hline CV $(\%)$ & & 24,0 & 23,7 & 15,8 & 6,5 & 7,4 & 18,0 \\
\hline
\end{tabular}

${ }^{(1)}$ Médias seguidas por letras iguais, nas colunas, não diferem entre si pelo teste de Tukey, a 5\% de probabilidade. 
um período mais tardio, o que resultou na proteção da cultura por um período mais longo.

$\mathrm{Na}$ AACPD, que sumariza as avaliações da severidade ao longo do ciclo, os menores valores foram observados nos tratamentos com trifloxistrobina + epoxiconazol, aplicados no início dos sintomas e com duas aplicações seqüenciais após 20 DAA1 (T3) ou somente uma aos 30 DAA1 (T5), e também aplicados a $1 \%$ de severidade da doença, com mais duas reaplicações seqüenciais em intervalos após 20 DAA1 (T4). A variável AACPD apresentou alta correlação com as avaliações de severidade realizadas em R5 $(\mathrm{r}=0,95)$ e R5.3 $(\mathrm{r}=0,97)$ e com a produtividade $(\mathrm{r}=-0,94)$. Nas avaliações de NDVI, realizadas em R5.3 e R5.4, os maiores valores foram observados nos mesmos tratamentos que apresentaram os menores valores de AACPD e maiores produtividades (T3, T4 e T5). A variável NDVI apresentou alta correlação com a produtividade, nas avaliações em R5.3 $(\mathrm{r}=0,97)$ e $\mathrm{R} 5.4(\mathrm{r}=0,98)$.

A baixa produtividade observada no experimento pode ser explicada pelo fato de a época de semeadura estar no limite do recomendado para essa variedade e pela alta intensidade da ferrugem. Mesmo nos tratamentos com três aplicações de fungicida, foi observada alta severidade de ferrugem em R5.3, o que evidencia que a calendarização não é a melhor maneira para se definirem as reaplicações. Esse mesmo resultado foi observado por Reis et al. (2007), que concluíram que o controle da FAS com base no estádio fenológico da cultura não deve ser realizado, pelo fato de o patógeno se estabelecer em qualquer estádio da planta. O monitoramento da FAS deve ser realizado para se definirem as primeiras aplicações e também as reaplicações.
$\mathrm{Na}$ terceira época de semeadura, o tratamento com piraclostrobina + epoxiconazol foi inferior em todas variáveis analisadas, quando comparado com o de trifloxistrobina + tebuconazol, aplicado nas mesmas épocas. Essa menor eficiência dos produtos piraclostrobina + epoxiconazol, em alta pressão da FAS, já foi observada em ensaios em rede, realizados em diferentes regiões produtoras (Godoy et al., 2007).

As avaliações de NDVI, realizadas com o equipamento Greenseeker, mostraram altas correlações com a severidade e com a produtividade, em todas as épocas de plantio. Análises por sensoriamento remoto têm sido utilizadas para avaliar doenças em outros patossistemas (Nutter Junior, 1989; Nicolas, 2004), mas não dispensam a avaliação de severidade, uma vez que outros fatores que causam estresse na cultura podem interferir nos valores de NDVI. A vantagem da avaliação de NDVI é a de que ela é rápida, precisa e evita a subjetividade.

O tratamento de semente com fluquinconazol não mostrou atraso na evolução da doença, conforme indicação em Tecnologias... (2008). No presente trabalho, foi realizada a avaliação do tratamento de semente com fluquinconazol, dentro de um sistema de manejo, conforme recomendado por Goulart (1999). A incidência inicial da ferrugem ocorreu em diferentes estádios fenológicos, mas não foi observada interação significativa, em nenhuma das épocas de semeaduras e variáveis analisadas.

Considerando-se a variação na incidência inicial da doença, em razão da influência do inóculo e das condições climáticas, observadas nesses ensaios e variáveis a cada safra, não se justifica a adoção de um modelo único para o manejo da doença, baseado em

Tabela 3. Severidade de ferrugem (\%) em R5 e R5.3, área abaixo da curva de progresso da doença (AACPD), índice vegetativo por diferença normalizada (NDVI) e produtividade $\left(\mathrm{kg} \mathrm{ha}^{-1}\right)$, dos diferentes tratamentos aplicados no início dos sintomas (I), com 1\% de severidade e com duas reaplicações em intervalos de 20 dias ou uma aplicação aos 30 dias após a primeira aplicação (DAA1), na cultivar BRS 247RR de ciclo médio, semeada em 13/12/2007(1).

\begin{tabular}{|c|c|c|c|c|c|c|c|}
\hline \multirow[t]{2}{*}{ Tratamento } & \multirow{2}{*}{$\begin{array}{c}\text { Momento } \\
\text { da aplicação }\end{array}$} & \multicolumn{2}{|c|}{ Severidade $(\%)$} & \multirow[t]{2}{*}{ AACPD } & \multicolumn{2}{|c|}{ NDVI } & \multirow{2}{*}{$\begin{array}{l}\text { Produtividade } \\
\qquad\left(\mathrm{kg} \mathrm{ha}^{-1}\right)\end{array}$} \\
\hline & & R5 & R5.3 & & R5.3 & R5.4 & \\
\hline 1. Testemunha & & $24,2 \mathrm{a}$ & $67,3 a$ & $1.236 \mathrm{a}$ & $0,73 \mathrm{c}$ & $0,56 \mathrm{~d}$ & $179 \mathrm{~d}$ \\
\hline 2. Piraclostrobina + epoxiconazol & $\mathrm{I} / 20 \mathrm{DAA} 1$ & $9,3 \mathrm{~b}$ & $41,3 b$ & $593 b$ & $0,81 b$ & $0,68 \mathrm{c}$ & $572 \mathrm{c}$ \\
\hline 3. Trifloxistrobina + tebuconazol & I/20 DAA 1 & $5,4 \mathrm{c}$ & $28,0 \mathrm{~d}$ & $415 c$ & $0,84 \mathrm{a}$ & $0,77 \mathrm{ab}$ & $836 \mathrm{ab}$ \\
\hline 4. Trifloxistrobina + tebuconazol & $1 \% / 20$ DAA 1 & $4,0 \mathrm{c}$ & $19,3 \mathrm{e}$ & $394 c$ & $0,85 \mathrm{a}$ & $0,78 \mathrm{a}$ & $1.031 \mathrm{a}$ \\
\hline 5. Trifloxistrobina + tebuconazol & I/30 DAA1 & $9,3 b$ & $28,0 \mathrm{~d}$ & $455 c$ & $0,83 \mathrm{ab}$ & $0,75 \mathrm{ab}$ & $871 \mathrm{a}$ \\
\hline 6. Trifloxistrobina + tebuconazol & $1 \% / 30$ DAA 1 & $5,5 \mathrm{c}$ & $34,7 \mathrm{c}$ & $597 b$ & $0,81 b$ & $0,71 \mathrm{bc}$ & $634 \mathrm{bc}$ \\
\hline $\mathrm{CV}(\%)$ & & 23 & 11,5 & 7,5 & 1,76 & 5,72 & 21,9 \\
\hline
\end{tabular}

${ }^{(1)}$ Médias seguidas por letras iguais, nas colunas, não diferem entre si pelo teste de Tukey, a 5\% de probabilidade. 
aplicações calendarizadas. O monitoramento da doença, na lavoura e na região, é importante para a definição das aplicações iniciais e deve ser realizado durante todo o ciclo, para se definirem as reaplicações e garantir melhor controle da doença e maior produtividade.

\section{Conclusões}

1. O tratamento de semente de soja com fluquinconazol não atrasa $o$ aparecimento dos primeiros sintomas nem a evolução da doença e não apresenta benefício no manejo da ferrugem.

2. As aplicações foliares de fungicidas, de forma calendarizada, não são eficientes para um controle satisfatório da ferrugem-da-soja.

\section{Agradecimentos}

A Maria Cristina Neves de Oliveira (equipe de biometria, bioinformática e socioeconomia) e a Tânia Fagundes, do Laboratório de Biometria da Embrapa Soja, pela orientação, interpretação e realização das análises estatísticas.

\section{Referências}

CAMPBELL, C.L.; MADDEN, L.V. Introduction to plant disease epidemiology. New York: John Willey, 1990. 532p.

GODOY, C.V.; CANTERI, M.G. Efeitos protetor, curativo e erradicante de fungicidas no controle da ferrugem-da-soja causada por Phakopsora pachyrhizi, em casa de vegetação. Fitopatologia Brasileira, v.29, p.97-101, 2004.

GODOY, C.V.; KOGA, L.J.; CANTERI, M.G. Diagrammatic scale for assessment of soybean rust severity. Fitopatologia Brasileira, v.31, p.63-68. 2006

GODOY,C.V.;PIMENTA,C.B.;MIGUEL-WRUCK,D.S.;RAMOS JUNIOR, E.U.; SIQUERI, F.V.; FEKSA, H.R.; SANTOS, I. dos; LOPES, I.O.N.; NUNES JUNIOR, J.; ITO, M.A.; IAMAMOTO, M.M.; ITO, M.F.; MEYER, M.C.; DIAS, M.D.; MARTINS, M.C.; ALMEIDA, N.S.; ANDRADE, N.S.; ANDRADE, P.J.M.; SOUZA, P.I.M.; BALARDIN, R.S.; BARROS, R.; SILVA, S.A. da; FURLAN, S.F.; GAVASSONI, W.L. Eficiência de fungicidas para controle da ferrugem-asiática-da-soja, Phakopsora pachyrhizi, na safra 2006/07: resultados sumarizados dos ensaios em rede. Londrina: Embrapa Soja, 2007. 8p. (Embrapa Soja. Circular técnica, 42). Disponível em: $<$ http://www.cnpso.embrapa. br/download/cirtec42.pdf $>$. Acesso em: 17 jul. 2008.

GOULART, A.C.P. Controle do oídio e da ferrugem-da-folha pelo tratamento de sementes de trigo com fungicidas. Dourados: Embrapa Agropecuária Oeste, 1999. 26p. (Embrapa Agropecuária
Oeste: Boletim de Pesquisa, 1). Disponível em: $<$ http://www.cpao. embrapa.br/publicacoes/ficha.php?tipo=BP\&num $=1 \&$ ano=1999>. Acesso em: 17 jul. 2008.

HENNING, A.A. Patologia e tratamento de sementes: noções gerais. Londrina: Embrapa Soja, 2005. 52p. (Embrapa Soja. Documentos, 264). Disponível em: $<$ http://www.cnpso.embrapa. br/download/alerta/documento264.pdf $>$. Acesso em: 17 jul. 2008.

LEVY, C. Epidemiology and chemical control of soybean rust in Southern Africa. Plant Disease, v.89, p.669-674, 2005.

MILES, M.R.; HARTMAN, G.L.; LEVY, C.; MOREL, W. Current status of soybean rust control by fungicides. Pesticide Outlook, v.14, p.197-200, 2003.

NICOLAS, H. Using remote sensing to determine the date of a fungicide application on winter wheat. Crop Protection, v.23, p.853-863, 2004.

NILSSON, H.E. Remote sensing and image analysis in plant pathology. Canadian Journal of Plant Pathology, v.17, p.154-166, 1995.

NUTTER JUNIOR, F.W. Detection and measurement of plant disease gradients in peanut with a multispectral radiometer. Phytopathology, v.79, p.958-963, 1989.

PICININI, E.C.; FERNANDES, J.M. Efeito do tratamento de sementes com fungicidas sobre o controle de doenças na parte aérea do trigo. Fitopatologia Brasileira, v.28, p.515-520, 2003.

REIS, E.F. dos; LIMA NETO, V. da C.; GODOY, C.V.; ROSA, C.T.; CASTANHO, H.E.; VICENTE, N.G. Controle químico da ferrugem-asiática-da-soja na região Sul do Paraná. Scientia Agraria, v.8, p.319-323, 2007.

RITCHIE, S.W.; HANWAY, J.J.; THOMPSON, H.E.; BENSON, G.O. How a soybean plant develops. Ames: Iowa State University of Science and Technology, 1985. 20p. (Special Report, n.53).

SAS INSTITUTE. SAS/STAT: versão 9.1.3. Cary: SAS Institute, 2001.

SINCLAIR, J.B.; HARTMAN, G.L. Soybean rust. In: HARTMAN, G.L.; SINCLAIR, J.B.; RUPE, J.C. (Ed.). Compendium of soybean diseases. $4^{\text {th }}$ ed. Saint Paul, MN: APS Press, 1999. p.25-26.

TECNOLOGIAS de produção de soja - Região Central do Brasil 2008. Londrina: Embrapa Soja: Planaltina: Embrapa Cerrados: Campinas: Embrapa Agropecuária Oeste, 2008. 280p. (Embrapa Soja. Sistema de Produção, 12).

YANG, X.B.; TSCHANZ, A.T.; DOWLER, W.M.; WANG, T.C. Development of yield loss models in relation to reductions of components of soybean infected with Phakopsora pachyrhizi. Phytopathology, v.81, p.1420-1426, 1991.

YORINORI, J.T.; PAIVA, W.M.; FREDERICK, R.D.; COSTAMILAN, L.M.; BERTAGNOLLI, P.F.; HARTMAN, G.E.; GODOY, C.V.; NUNES JUNIOR, J. Epidemics of soybean rust (Phakopsora pachyrhizi) in Brazil and Paraguay from 2001 to 2003. Plant Disease, v.89, p. 675-677, 2005. 